Pacific Journal of Mathematic 


\section{FUNCTIONS ACTING IN WEIGHTED ORLICZ ALGEBRAS}

\section{Horst BEHNCKE}

Any complex valued function $F$ with $F(0)=0$, which is Lipschitz continuous at 0 operates on all weighted Orlicz sequence algebras. If the weight increases sufficiently rapidly the class of functions which operate is strictly larger than the above class.

In this note we investigate the functional calculus of weighted Orlicz sequence algebras. We show that such an algebra has non Lipschitz continuous functions operating on it, if the weight increases sufficiently rapidly. On the other hand one knows: Let $\mathscr{A}$ be a commutative semisimple complex completely regular Banach algebra with identity and hermitian involution. Assume a function $F:(-1,1) \rightarrow \mathbf{R}$ with $F(0)=0$ and $\lim _{t \rightarrow 0}|F(t) / t|=\infty$ operates on $\mathscr{A}$. Then $\mathscr{A}$ is the algebra of all continuous functions on its spectrum [1, Corollary 8.5]. This note was motivated by a paper of F. Gulick [2], who investigated the functional calculus of commutative ${ }_{*}$-subalgebras of $\mathscr{C}_{\dot{p}}(\mathscr{H}), 1 \leqq p<\infty$, the algebra of all compact operators $x$ on some Hilbert space $\mathscr{H}$ with $|x|_{p}=$ $\left(\operatorname{Tr}\left(x^{*} x\right)^{p / 2}\right)^{1 / p}<\infty$.

Let $\mathscr{A}$ be a commutative $*$-subalgebra of $\mathscr{C}_{p}(\mathscr{H}), 1 \leqq p<\infty$, for some Hilbert space $\mathscr{H}$. By the spectral theorem the elements of $\mathscr{A}$ can be diagonalized simultaneously, i.e. there exists a sequence of finite dimensional projections $\left(P_{i}\right)_{i \in l}$, such that each $x \in \mathscr{A}$ can be written as $x=\sum \lambda_{i}(x) P_{\imath}$ and $|x|=\left(\operatorname{Tr}\left(x^{*} x\right)^{p / 2}\right)^{1 / p}=\left(\sum\left|\lambda_{i}(x)\right|^{p} \operatorname{dim} P_{i}\right)^{1 / p}$. Clearly the spectrum of $\mathscr{A}$ can be identified with $I$. The Gelfand representation of $\mathscr{A}$ leads then to the following class of Banach algebras. Let $I$ be a set and $e$ a real valued function on $I$ with $e(i) \geqq 1$, the weight function. Let $\mathscr{A}=l_{p, e}(I), 1 \leqq p<\infty$ be the system of all complex (real) valued functions $x$ on $I$ with $|x|_{p}=\left(\sum|x(i)|^{p} e(i)\right)^{1 / p}<\infty$. Such an algebra one may call a weighted $l^{p}$-algebra. Hence in [2] Gulick actually studied the functional calculus of weighted $l^{p}$-algebras. It is natural to investigate the problem of the functional calculus in the context of the larger class of Orlicz sequence algebras, since it is essentially determined by the weight (Lemma 4) and depends to a lesser extent on the Orlicz function. Our results are considerable extensions of those in $[2, \S 5]$, even in the case of weighted $l^{p}$-algebras.

Let $M$ be a continuous nondecreasing convex function on $[0, \infty)$ with 


$$
M(0)=0, \quad M(x)>0 \text { for } x>0 \text { and } M(\infty)=\infty .
$$

We may normalize $M$ by

$$
M(1)=1
$$

because of $\{3$, Prop. 1.4.2]. Moreover we shall assume that $M$ satisfies the $\Delta_{2}$-condition at $0[3$, prop. 1.4.1] i.e.

$$
\lim _{x \rightarrow 0+} \sup M(2 x) / M(x)<\infty .
$$

It suffices to require the $\Delta_{2}$ condition at 0 only, because we only study algebras of sequences. Because of (3) we may moreover require $M$ to be continuously differentiable, [3, Remark on p. 68]. A function $M$ satisfying these requirements we shall call an Orlicz function henceforth. Let $M$ be an Orlicz function. Then $M^{\prime}$ is increasing. This and $M(x)=\int_{0}^{x} M^{\prime}(t) d t$ yields immediately

$$
M(k x) \geqq k M(x) \text { for } k \geqq 1 \text {. }
$$

Later we shall also need

(5) $\lim M^{-1}\left(h_{n} r_{n}\right) / M^{-1}\left(r_{n}\right)=\infty$ for each sequence $r_{n} \searrow 0$ and $h_{n} \nearrow \infty$.

By $r_{n} \searrow 0$ we mean that $r_{n}$ is a sequence of positive numbers with $r_{n}>r_{n+1}$ and $\lim r_{n}=0$.

If (5) were not true, choose a subsequence $\left\{r_{n_{k}}\right\}$ with $M^{-1}\left(h_{n_{k}} r_{n_{k}}\right)=$ $s_{k} M^{-1}\left(r_{n_{k}}\right)$ and $s_{k} \leqq K$. To this apply $M$ and set $y_{k}=M^{-1}\left(r_{n_{k}}\right)$. We get $h_{n k} M\left(y_{k}\right) \leqq M\left(K y_{k}\right) \leqq K^{\prime} M\left(y_{k}\right)$ by (3), a contradiction.

Let $M$ be an Orlicz function and let $I$ be a set. Further let $e$ be a real valued function on $I$ with $e(i) \geqq 1$. For a complex valued function $x$ on $I$ we can then define

$$
\phi_{M, e}(x)=\sum M(|x(i)|) e(i)
$$

If such a function $x$ satisfies $\phi_{M, e}(x)<\infty$ one also has $\phi_{M . e}(\lambda x)<\infty$ for all complex $\lambda$ because of (3). Similarly one shows with (3) that $\phi_{M, e}(x), \phi_{M, e}(y)<\infty$ implies $\phi_{M, e}(x+y)<\infty$. Hence the set $l_{M, e}(I)$ of all complex (real) valued functions $x$ with $\phi_{M, e}(x)<\infty$ is a linear space. It can be normed by

$$
\|x\|=\inf \left\{t \mid \phi_{M, e}\left(\frac{1}{t} x\right) \leqq 1\right\}
$$


Let $x, y \in l_{M, e}(I)$ with $\|x\| \leqq 1$ and $\|y\| \leqq 1$. Then $|x(i)| \leqq 1$ for all $i$ and $\phi_{M, e}(x \cdot y)=\sum M(|x(i) y(i)|) e(i) \leqq \sum M(|y(i)|) e(i) \leqq 1$ or $\|x \cdot y\| \leqq 1$. Thus $l_{M, e}(I)$ is even an algebra.

Lemma 1. Let $M$, e and $\mathscr{A}=l_{M, e}(I)$ be as above. Then $\mathscr{A}$ is a complex (real) semisimple Banach algebra with spectrum I. It is separable iff I is countable.

The proof of this lemma is rather easy and therefore omitted. Such an algebra $\mathscr{A}=l_{M, e}(I)$ we shall call a weighted Orlicz sequence algebra with weight $e$ and Orlicz function $M$. Since any $x \in \mathscr{A}$ satisfies: supp $x$ is countable and $x(i) \rightarrow 0$ as $i \rightarrow \infty$, the algebra $\mathscr{A}$ is generated by its minimal projections. This holds also for each closed subalgebra $\mathscr{B}$ of $\mathscr{A}$. Let $\mathscr{B} \subset \mathscr{A}$ be such a subalgebra of $\mathscr{A}$ and let $\left\{p_{\jmath}\right\}_{, \in J}$ be its system of minimal idempotents. Then $p_{j}$ is the characteristic function of some finite subset $I_{j} \subset I$. Hence we can write $I=I_{0} \dot{\cup} \dot{U}_{j \in J} I_{j}$, where $\dot{U}$ denotes disjoint union. Thus $\mathscr{B}$ is of the form $l_{M, f}(J)$ with $f(j)=$ $\sum_{\iota \in I_{1}} e(i)$.

Let $S$ be an open set in $\mathbf{R}$ or $\mathbf{C}$ and let $F$ be a real or complex valued function defined on $S$. We say that $F$ operates on the function algebra $\mathscr{A}$, if $F \circ a \in \mathscr{A}$ for each $a \in \mathscr{A}$ with range in $S$. The set of all functions operating on $\mathscr{A}$ defines the functional calculus of $\mathscr{A}$.

For the remainder of this paper we shall fix $M$ and the weight $e$ and consider only complex algebras, though the results are true also in the real case. Since $l_{M, e}(I)$ has the functional calculus of all complex functions, if $I$ is finite, we shall assume from now on that $I$ is infinite. Regarding the functional calculus of $\mathscr{A}=l_{M, e}(I)$ one can easily show.

Lemma 2. (i) Let $F$ operate on $\mathscr{A}$ then $F$ is continuous at 0 and $F(0)=0$.

(ii) Let $\mathscr{L}_{0}$ be the class of all functions $F$ with $F(0)=0$, which are Lipschitz continuous at 0 , i.e. $|F(z)| \leqq K_{F}|z|$ for $0<|z| \leqq \delta_{F}$ for some $\delta_{F}>0$ and $K_{F}>0$. Then $\mathscr{L}_{0}$ operates on $\mathscr{A}$.

(iii) If $F$ operates on $\mathscr{A}$ also the absolute value of $F$ operates on $\mathscr{A}$.

(iv) If $F$ operates on $\mathscr{A}$ and if $G$ is a function with $|G(z)| \leqq|F(z)|$ for all $z$, then also $G$ operates on $\mathscr{A}$.

Assume a function $F \notin \mathscr{L}_{0}$ operates on $\mathscr{A}$. Then there exist $z_{n} \in \boldsymbol{C}$ with $\left|z_{n}\right| \searrow 0$, such that $\left|F\left(z_{n}\right) / z_{n}\right| \geqq n$. Because of Lemma 2 (iv) also the function

$$
G(z)= \begin{cases}n z_{n} & z=z_{n} \\ 0 & \text { otherwise }\end{cases}
$$


operates on $\mathscr{A}$. Then also the function $H$ with

$$
H(z)= \begin{cases}n\left|z_{n}\right| & z=\left|z_{n}\right| \\ 0 & \text { otherwise }\end{cases}
$$

operates on $\mathscr{A}$. Hence we may assume without loss of generality that $F$ has the form

$$
F(z)= \begin{cases}n z_{n} & z=z_{n} \\ 0 & \text { otherwise }\end{cases}
$$

for some sequence $z_{n}$ with $z_{n} \searrow 0$. Let $\left\{n_{k}\right\}$ be a strictly increasing sequence of natural numbers. By Lemma 2 (iv) also the function $G$ with

$$
G(z)= \begin{cases}k z_{n k} & z=z_{n k} \\ 0 & \text { otherwise }\end{cases}
$$

operates on $\mathscr{A}$. Since we shall use this technique quite often in the sequel we say that $G$ is obtained from $F$ by pruning. Though the functions $F(8)$ are rather discontinuous, the continuity of functions operating in $\mathscr{A}$ is not an essential condition, with the exception of continuity at 0 .

Lemma 3. Assume a function $F \notin \mathscr{L}_{0}$ operates on $\mathscr{A}$. Then there exists a continuous $G \notin \mathscr{L}_{0}$ operating on $\mathscr{A}$.

Proof. We may assume that $F$ has the form (8). Now let $\delta_{n}=$ $\frac{1}{4} \min \left(z_{n}-z_{n+1}, z_{n-1}-z_{n}\right)$ and define the function $G$ by

$$
G(z)= \begin{cases}n z_{n} & z=z_{n} \\ \text { linear } & \text { on } I_{n}=\left(z_{n}-\delta_{n}, z_{n}+\delta_{n}\right) \\ 0 & \text { otherwise. }\end{cases}
$$

Then $G$ is continuous and $G \notin \mathscr{L}_{0}$. Let $x \in \mathscr{A}$. We want to show $G x \in \mathscr{A}$. For this it suffices to assume $x(i)=0$ or $x(i) \in \cup I_{n}$. Now define $y$ by $y(i)=0$ if $x(i)=0$ and $y(i)=z_{n}$ if $x(i) \in I_{n}$. Then $\frac{1}{2} x(i) \leqq$ $y(i) \leqq 2 x(i)$ and $y \in \mathscr{A}$. By assumption $G y=F y \in \mathscr{A}$. Since $(G x)(i) \leqq(G y)(i)$ we see $G x \in \mathscr{A}$.

By $l_{p, e}(I), 1 \leqq p<\infty$, we shall denote the algebra $l_{M, e}(I)$ with $M=x^{p}$.

LEMMA 4. $\mathscr{A}=l_{\text {M.e }}(I)$ has the functional calculus of $\mathscr{L}_{0}$ only iff $l_{1, e}(I)$ has only $\mathscr{L}_{0}$ operating on it. 
Proof. (a) Assume a function $F \notin \mathscr{L}_{0}$ operates on $\mathscr{A}$. We may take $F$ to be of the form (8). Then the function $G$ with

$$
G(z)= \begin{cases}M\left(n z_{n}\right) & z=M\left(z_{n}\right) \\ 0 & \text { otherwise }\end{cases}
$$

operates on $l_{1, e}(I)$. By (4) $G \notin \mathscr{L}_{0}$.

(b) For the converse replace $M$ by $M^{-1}$ and use (5). This lemma will simplify our computations considerably.

LemmA 5. (i) Let $J \subset I$ and $\mathscr{A}(J)=\{x \in \mathscr{A} \mid$ support $x \in J\}$ then $\mathscr{A}(J)$ is an ideal and each function operating on $\mathscr{A}$ also operates on $\mathscr{A}(J)$.

(ii) Let $e$ and $f$ be weight functions with $(1 / K) e \leqq f \leqq K e$ for some $K>0$. Then $l_{M, e}(I)=l_{M, f}(I)$ and both norms are equivalent. In particular both algebras have the same functional calculus.

(iii) If $e$ is bounded $l_{M, e}(I)$ has only the functional calculus of $\mathscr{L}_{0}$.

(iv) Every nonseparable $l_{M, e}(I)$ has only the functional calculus of $\mathscr{L}_{0}$.

Proof. (i) and (ii) are trivial (see also [3], p. 68) (iii) has been shown by Gulick [2] for $l_{p, e}(N)$ but this proof extends easily to this case. It is also possible to adapt the proof of Theorem 1 below to this situation.

(iv) Since $e(i) \geqq 1$ and because of (ii) we can replace $e$ by an equivalent weight function with integer values only. Hence there is not loss of generality to assume that $e$ has only integer values. If $l_{M, e}(I)$ is nonseparable $I$ is uncountable. Now let $I_{n}=\{i \in I \mid e(i)=n\}$. Then $I=\cup I_{n}$ and some $I_{n o}$ must be infinite. By (iii) only $\mathscr{L}_{0}$ acts on $\mathscr{A}\left(I_{n_{0}}\right)$, hence by (i) only $\mathscr{L}_{0}$ operates on $\mathscr{A}$.

Because of this lemma we shall assume from now on:

$I$ is countably infinite.

$e$ is integer valued.

Each $I_{n}=e^{-1}(n)$ is finite,

since otherwise $\mathscr{A}$ has only the functional calculus of $\mathscr{L}_{0}$. Thus we may identify $I$ with $\mathbf{N}$. Moreover this identification may be constructed such that the sets $I_{1}, I_{2}, \cdots$ are mapped onto successive intervals on $\mathbf{N}$. In this way $e$ is mapped onto an increasing function on $\mathbf{N}$ with values in $\mathbf{N}$. For the remainder we shall make these assumptions on $I$ and $e$ and denote $l_{M, e}(\mathbf{N})$ by $l_{M, e}$.

Let $e_{1}, e_{2}, \cdots$ be the values of $e$ in increasing order. By assumption there exist a strictly increasing sequence $n_{\imath}$ of natural numbers with

$$
e(n)=e_{k} \quad \text { for } \quad n_{k-1} \leqq n<n_{k}, \quad k=1, \cdots
$$

where $n_{0}=1$. 
The number $m_{k}=n_{k}-n_{k-1}$ we shall call the multiplicity of $e_{k}$. Clearly the set $\left\{\left(e_{k}, m_{k}\right)\right\}$ determines $e$ uniquely. For this we write $e \sim\left\{\left(e_{k}, m_{k}\right)\right\}$.

Lemma 6. (i) Let $e \sim\left\{\left(e_{k}, m_{k}\right)\right\}$ and $f \sim\left\{\left(e_{k}, l_{k}\right)\right\}$. If only $\mathscr{L}_{0}$ operates on $l_{M, e}$ and $m_{k} \leqq l_{k}$ for all $k$, only $\mathscr{L}_{0}$ operates on $l_{M, f}$.

(ii) If $K^{-1} \leqq m_{k} / l_{k} \leqq K$ for some $K>0$ and all $k$, then $l_{M, e}$ and $l_{M, f}$ have the same functional calculus.

Proof. (i) Follows immediately from Lemma 5(i).

(ii) We may assume $m_{k} \leqq l_{k}$, otherwise we use $\left\{\left(e_{k}, \min \left(m_{k}, l_{k}\right)\right\}\right.$ as an intermediate weight. By Lemma 5(i) any $F$ operating on $l_{M, f}$ also operates on $l_{M, e}$. Conversely let $F$ operate on $l_{M, e}$ and let $x \in l_{M, f}$. Since $l_{k} / m_{k} \leqq K$ we can write $x=x_{1}+\cdots+x_{r}$ with $x_{i} \cdot x_{j}=0$ for $i \neq j$ and supp $x_{i} \cap e^{-1}\left(e_{n}\right)$ has less than $m_{n}$ elements. Then $F x=F x_{1}+\cdots+$ $F x_{r} \in l_{M, f}$ since each $x_{i}$ belongs to a subalgebra of $l_{M, f}$, which is of the form $l_{M, e}$.

With these preparations we can now show

THEOREM 1. Let $e \sim\left\{\left(e_{k}, m_{k}\right)\right\}$ be a weight. Then $\mathscr{A}=l_{\text {M.e }}$ has only the functional calculus of $\mathscr{L}_{0}$, if these exist a constant $K>0$ with $e_{k+1} / e_{k} \leqq K$ for all $k$.

Proof. The proof is indirect and divided into two steps. In the first step we modify the weight and the function and in the second we construct a suitable $x \in \mathscr{A}$.

(a) Without loss of generality, we may assume $\mathscr{A}=l_{1, e}$ with $m_{k}=1$ for all $k$ (Lemma 5,6). Assume a function $F \notin \mathscr{L}_{0}$ operates on $\mathscr{A}$. We may take $F$ to be of the form (8) for some positive sequence $z_{n} \searrow 0$ with $z_{n} / z_{n+1} \geqq 2 K$ even. Since $e_{k+1} / e_{k} \leqq K$ for all $k$, there exist a strictly increasing sequence of natural numbers $l(n)$ with $1 \leqq z_{n} \cdot e_{l(n)}^{+1} \leqq$ $K$. Then also the function $F$ with $z_{n}=e_{l(n)}^{-1}$ operates on $\mathscr{A}$. By using the pruning procedure if necessary we can even achieve $l(n) \geqq n^{2}$ and $e_{l(n+1)} / e_{l(n)} \geqq n^{3}$.

(b) Since $e_{k+1} / e_{k} \leqq K$ there exists for each $n \geqq K$ a natural number $r(n)$ with $l(n) \leqq r(n)<l(n+1)$ and

$$
\frac{1}{K n^{2}} \leqq e_{r(n)} / e_{l(n+1)} \leqq \frac{1}{n^{2}} .
$$

If we choose $r(n)$ maximal with this property, $r(n)$ is even unique. Now let $x \in \mathscr{A}$ be defined by

$$
x(m)= \begin{cases}e_{l(n+1)}^{-1} & m=r(n), \quad n=1,2, \cdots \\ 0 & \text { otherwise. }\end{cases}
$$


Then $|x|_{1}=\sum e_{r(n)} e_{l(n+1)}^{-1}<\infty$ but $|F x|_{1}=\sum(n+1) e_{r(n)} e_{l(n+1)}^{-1}=\infty$, which gives the desired contradiction.

This result is a considerable extension of Theorem 5.4 of [2].

Above we had already considered the normalization of the weight $e$. This normalization can be carried further. In fact one can easily show that for any weight $e$ and constant $K>1$ there exist a weight $f \sim\left\{\left(f_{k}, l_{k}\right)\right\}$ with $(1 / 2 K) f<e<2 K f$ and $f_{k+1} / f_{k} \geqq K$ for all $k$.

THEOREM 2. Let $e \sim\left\{\left(e_{k}, m_{k}\right)\right\}$ be a weight and assume $e_{k+1} / e_{k} \geqq$ $K>1$ and $m_{k} e_{k} \cdot e_{k+1}^{-1} \leqq K^{\prime}$ for some $K$ and $K^{\prime}$ for all $k$ and $\liminf m_{k} e_{k} e_{k+1}^{-1}=0$. Then there exists a continuous but not Lipschitz continuous function $F$ operating on $\mathscr{A}=l_{M, e}$.

Proof. Define inductively a sequence $r_{n}$ of natural numbers by

(i) $\quad r_{0}=1$

(ii) $r_{n+1}>r_{n}$

(iii) $m_{r_{n}-1} e_{r_{n}-1} \cdot e_{r_{n}}^{-1} \leqq 2^{-n}$.

If $r_{n}$ is defined in this fashion, let for each $l \in \mathbf{N} p_{l}$ denote the smallest number in $\left\{r_{n} \mid n \in \mathbf{N}\right\} \cap\{l+1, l+2, \cdots\}$. Then the series

$$
\sum_{l=1}^{\infty} m_{l} e_{l} e_{p_{l}}^{-1}=\sum_{k=0}^{\infty} \sum_{l=r_{k}}^{r_{k+1}-1} m_{l} e_{l} e_{r_{k+1}}^{-1}
$$

converges, since for $r_{k} \leqq l<r_{k+1}$ we have the estimate

$$
\begin{aligned}
m_{l} \cdot e_{l} e_{r_{k+1}}^{-1} & =\left(m_{l} e_{l} e_{l+1}^{-1}\right)\left(e_{l+1} e_{l+2}^{-1}\right) \cdots\left(e_{r_{k+1}-1} e_{r_{k+1}}^{-1}\right) \\
& \leqq K^{\prime} K^{-\left(r_{k+1}-l-2\right)} 2^{-k}
\end{aligned}
$$

Hence there is a positive sequence $h_{l} \nearrow \infty$ such that $\sum_{l=1}^{\infty} m_{l} h_{l} e_{l} e_{p_{l}}^{-1}$ converges and such that

$$
\frac{e_{n+1}}{e_{n}} \geqq \frac{h_{n+1}}{h_{n}} \quad n=1,2, \cdots ; \quad h_{1}=1
$$

(b) With $\left\{r_{n}\right\}$ and $\left\{h_{n}\right\}$ determined as above let now $F$ be defined by

$$
F(z)= \begin{cases}h_{r_{n}} \cdot e_{r_{n}}^{-1} & \text { if } z=e_{r_{n}}^{-1} \\ 0 & \text { otherwise }\end{cases}
$$

then $F \notin \mathscr{L}_{0}$. If $F$ operates on $\mathscr{A}=l_{1, e}$, there exists also a continuous, not Lipschitz continuous, function operating on $\mathscr{A}$. Now let $x=$ 
$\left(x_{n}\right) \in l_{1, e}$. We want to show $F x \in l_{1, e}$. For this it suffices to assume that $x_{n} \in \operatorname{supp} F \cup\{0\}$. Hence we may write $x_{n}=\epsilon_{n} \cdot e_{r_{(n)}}^{-1}$ with $\epsilon_{n}=0,1$.

Then we have

$$
|x|_{1}=\sum_{l=1}^{\infty} \sum_{n=n_{l-1}}^{n_{l}-1} \epsilon_{n} \cdot e_{n(n)}^{-1} e_{l}<\infty .
$$

This is only possible if $\epsilon_{n} \neq 0$ implies $r_{l(n)}>l$ for almost all $n$ with $n_{l-1} \leqq n<n_{l}$. Neglecting this exceptional set, we may thus assume $r_{l(n)}>l$ for all $n_{l-1} \leqq n<n_{l}$. Then however we can estimate

$$
|x|_{1} \leqq \sum_{l=1}^{\infty} \sum_{n=n_{l-1}}^{n_{l}-1} \epsilon_{n} e_{p_{l}}^{-1} e_{l} \leqq \sum_{l=1}^{\infty} m_{l} e_{l} e_{p_{l}}^{-1}<\infty .
$$

Moreover we have

$$
\begin{aligned}
|F x|_{1} & =\sum_{l=1}^{\infty} \sum_{n=n_{l-1}}^{n_{l}-1} \epsilon_{n} h_{r(n)} e_{r_{l(n)}}^{-1} e_{l} \\
& =\sum_{l=1}^{\infty} \sum_{n=n_{l-1}}^{n_{l}-1} \epsilon_{n} \frac{h_{r_{l n}(n)}}{h_{p_{l}}} \cdot \frac{e_{p_{l}}}{e_{r_{l(n)}}} \cdot h_{p_{l}} e_{p_{l}}^{-1} e_{l} \\
& \leqq \sum_{l=1}^{\infty} \sum_{n=n_{l-1}}^{n_{l}-1} \epsilon h_{p_{l}} e_{p_{l}}^{-1} e_{l} \quad \text { by } \quad(10) \\
& \leqq \sum_{l=1}^{\infty} m_{l} h_{p_{l}} e_{p_{l}}^{-1} e_{l}<\infty .
\end{aligned}
$$

Hence $F x \in l_{1, e}$ and the proof is completed.

Theorem 2 shows that the results in [1, Chapter 8] are close to optimal. In fact the algebra $l_{M, e}$ with identity adjoined is a normal conjugate closed function algebra on which a continuous non Lipschitz continuous function operates. (I thank the referee for pointing this out.)

This result also covers the last example in [2]. The conditions of the theorem are not necessary for an $F \notin \mathscr{L}_{0}$ to operate. For the proof one only needs the existence of a sequence $r_{n}$ such that $\sum m_{k} e_{k} e_{p_{k}}^{-1}<$ $\infty$. Nevertheless Theorem 2 is close to optimal, because

THEOREM 3. Let $e \sim\left\{\left(e_{k}, m_{k}\right)\right\}$ with $e_{k} e_{k+1}^{-1} \rightarrow 0$ and $m_{k} e_{k} e_{k+1}^{-1} \geqq \epsilon>$ 0 . Then only $\mathscr{L}_{0}$ operates on $l_{\text {M.e }}, 1 \leqq p<\infty$.

Proof. (a) Because of Lemma 6 (ii), we may take $\epsilon=1$ and because of Lemma 4 we may take $\mathscr{A}=l_{1, e}$. Assume $F$ of the form (8) operates on $\mathscr{A}$. Now we have to distinguish two cases regarding $F$. 
(b) Assume there exist a $K>1$ and subsequences $l(n)$ and $k(n)$ of natural numbers, such that $K^{-1} \leqq e_{l(n)} z_{k(n)} \leqq K$, then the function

$$
G(x)= \begin{cases}n e_{l(n)}^{-1} & x=e_{l(n)}^{-1} \\ 0 & \text { otherwise }\end{cases}
$$

operates on $\mathscr{A}$. $G$ can be obtained from $K \cdot F$ by pruning. Applying the pruning procedure again if necessary, we may assume in addition that $e_{l(n)-1} \cdot e_{l(n)}^{-1} \leqq 2^{-n}$. Now define

$$
x(m)=\left\{\begin{array}{lll}
e_{l(n)}^{-1} & \text { if } & n_{l(n)-1} \leqq m \leqq n_{l(n)-1}+q(n) \\
0 & \text { if } & n_{l(n)-1}+q(n)+1 \leqq m<n_{l(n)}-1
\end{array}\right.
$$

where $q_{n}$ is a natural number with $q_{n} \leqq m_{l(n)-1}$ such that $q_{n} \cdot e_{l(n)}^{-1} e_{l(n)-1}$ is approximately $1 / n^{2}$ with an error of less than $2^{-n}$. By our assumptions this is possible. Clearly $x \in \mathscr{A}$, but $F x \notin \mathscr{A}$ a contradiction.

(c) Assume now $F$ as above. For every $z_{n}$, there exists a natural number $t(n)$ with $e_{t(n)+1}^{-1} \leqq z_{n}<e_{t(n)}^{-1}$. By applying the pruning procedure again, if necessary, we may assume even that each interval $\left[e_{i(n)+1}^{-1}, e_{t(n)}^{-1}\right)$ contains only $z_{n}$. Because of (b) we may assume that $\left\{z_{n} e_{t(n)+1}\right\}$ and $\left\{z_{n}^{-1} e_{t(n)}^{-1}\right\}$ contain no bounded subsequences. Hence in particular $z_{n}^{-1} e_{t(n)}^{-1}$ diverges. By using the pruning procedure again if necessary we may even assume $z_{n} e_{t(n)} \leqq 2^{-n}$. Now define

$$
x(m)=\left\{\begin{array}{lll}
z_{n} & \text { if } & n_{t(n)} \leqq m<n_{t(n)}+q_{n} \\
0 & \text { if } & n_{t(n)}+q_{n} \leqq m \leqq n_{t(n)+1}-1
\end{array}\right.
$$

where the natural number $q_{n}$ is chosen such that $q_{n} z_{n} e_{t_{n}}$ is approximately $1 / n^{2}$ with an error of less than $2^{-n}$. Because $e_{t(n)+1}^{-1}<z_{n}$ and $e_{t(n)+1}^{-1} e_{t(n)} m_{t(n)} \geqq 1$ this is possible. Then $x \in \mathscr{A}$ but $F x \notin \mathscr{A}$.

\section{REFERENCES}

1. R. B. Burckel, Characterization of $C(X)$ among its subalgebras, Lecture Notes in Pure and Applied Math. New York 1972.

2. F. Gulick, Actions of functions in Banach Algebras, Pacific J. Math., 34 (1970), 657-673.

3. J. Lindenstrauss and L. Tzafriri, Classical Banach Spaces, Springer Lecture Notes Vol. 338.

Received October 21, 1976 and in revised form March 29, 1977. 




\section{Pacific Journal of Mathematics \\ Vol. 70, No. $1 \quad$ September, 1977}

William H. Barker, Noether's theorem for plane domains with hyperelliptic

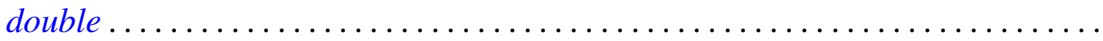

Michael James Beeson, Non-continuous dependence of surfaces of least area on the boundary curve ...................................... 11

Horst Behncke, Functions acting in weighted Orlicz algebras . . . . . . . . . . . . 19

Howard Edwin Bell, A commutativity study for periodic rings . . . . . . . . . . . 29

Peter Botta and Stephen J. Pierce, The preservers of any orthogonal group ....... 37

Douglas S. Bridges, The constructive Radon-Nikodým theorem ............. 51

James Dennis Brom, The theory of almost periodic functions in constructive

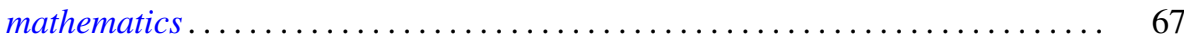

N. Burgoyne and C. Williamson, Semi-simple classes in Chevalley type groups ....

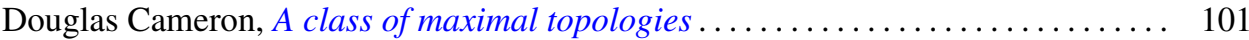

L. Carlitz, Enumeration of doubly up-down permutations . . . . . . . . . . . . . . 105

Paul Robert Chernoff, The quantum n-body problem and a theorem of

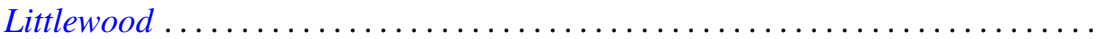

Jo-Ann Deborah Cohen, Locally bounded topologies on $F(X) \ldots \ldots \ldots \ldots \ldots \ldots$

Heinz Otto Cordes and Robert Colman McOwen, Remarks on singular elliptic

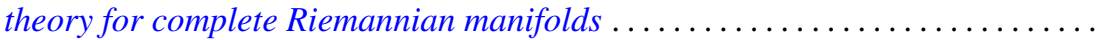

Micheal Neal Dyer, Correction to: "Rational homology and Whitehead

products"

Robert Fernholz, Factorization of Radonifying transformations

Lawrence Arthur Fialkow, A note on quasisimilarity. II ...... . .

Harvey Charles Greenwald, Lipschitz spaces of distributions on the surface of unit

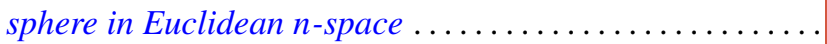

Albrecht Irle, On the measurability of conditional expectations

Tom (Roy Thomas Jr.) Jacob, Matrix transformations involving simple sequence

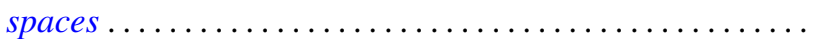

A. Katsaras, Continuous linear maps positive on increasing continuous

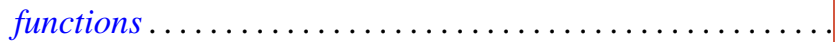

Kenneth Kunen and Judith Roitman, Attaining the spread at cardinals of cofinality

Lawrence Louis Larmore and Robert David Rigdon, Enumerating normal bundles

of immersions and embeddings of projective spaces ...... . .

Ch. G. Philos and V. A. Staïkos, Asymptotic properties of nonoscillatory solutions of differential equations with deviating argument .

Peter Michael Rosenthal and Ahmed Ramzy Sourour, On operator algebras containing cyclic Boolean algebras...

Polychronis Strantzalos, Strikt fast gleichgradig-stetige und eigentliche

Aktionen ...

Glenn Francis Webb, Exponential representation of solutions to an abstract

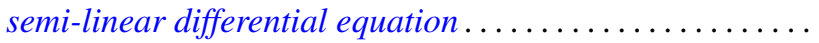

